\title{
List of Those Attending the Symposium
}

Adler, Helmut E., American Museum of Natural History, New York City

Akey, Alice, Adelphi College, Garden City, New York

Alexander, Mary L., University of Texas, Houston, Texas

Anderson, PaUl, Columbia University, New York City

Aschoff, Jürgen, Max-Planck-Institut für Verhaltensphysiologie, Erling-Andechs/Obb., Germany

Ball, Harold J., University of Nebraska, Lincoln, Nebraska

BarLow, JoHn S., Massachusetts Institute of Technology, Cambridge, Massachusetts and Massachusetts General Hospital, Boston, Massachusetts

BARNETr, AUdREY, Indiana University, Bloomington, Indiana

Barnwell, Franklin H., Northwestern University, Evanston, Illinois

Bartell, Clelmer K., Duke University, Durham, North Carolina

Barth, Robert, Harvard University, Cambridge, Massachusetts

Barton, Alexander, The Stony Brook School, Stony Brook, New York

Bennetr, Miriam F., Sweet Briar College, Sweet Briar, Virginia

Bianchi, Carla, Northwestern University, Evanston, Illinois

Birukow, Georg, Zoologisches Institut der Universität, Göttingen, Germany

Blakeslee, Alton, The Associated Press, New York City

Buiss, Dorothy E., The American Museum of Natural History, New York City

Braemer, Wolfgang, Max-Planck-Institut, Seewiesen, über Starnberg/Obb., Germany

Brandt, William H., Oregon State College, Corvallis, Oregon

Brehme, Katherine, Adelphi College, Garden City, New York

Brows, Clifford, National Film Board of Canada, Montreal, Canada

Brown, Frank A., JR., Northwestern University, Evanston, Illinois

Bruce, Victor G., Princeton University, Princeton, New Jersey

BüNNıNG, ERwIN, Botanisches Institut, Universität Tübingen, Germany

Bünsow, Roвert C., Pflanzenphysiologisches Institut der Universität, Göttingen, Germany

Butner, Alfred, Jamaica, New York

CARR, Archie, University of Florida, Gainesville, Florida
Cavanagh, James, Garden City, New York

Chovnick, Arthur, Biological Laboratory, Cold Spring Harbor, New York

Cloudsley-Thompson, J. L., University of London, King's College, London, England

Coluins, Walter, Colorado State University, Fort Collins, Colorado

Conklin, Marie E., Adelphi College, Garden City, New York

Corbet, Philip S., East Africa Virus Research Institute, Entebbe, Uganda, East Africa

DeCoursey, Patricia, University of Wisconsin, Madison, Wisconsin

Ehret, Charles F., Argonne National Laboratory, Lemont, Illinois

Engelmann, Franz, Albert Einstein College of Medicine, New York City

ENright, J. T., University of California, LaJolla, California

Fay, Anthony F., Flushing, New York

Fingerman, Milton, Tulane University, New Orleans, Louisiana

Flynn, John E., Office of Naval Research, New York City

FolK, G. Edgar, State University of Iowa, Iowa City, Iowa

Frisch, Leonora, Biological Laboratory, Cold Spring Harbor, New York

Galinsky, Inving, Hofstra College, Hempstead, New York

Galler, S. R., Office of Naval Research, Washington, D. C.

Gay, Helen, Carnegie Institution of Washington, Cold Spring Harbor, New York

Gould, EDwin, Tulane University, New Orleans, Louisiana

Grace, Edwin J., Grace Clinic Group, Brooklyn, New York

Griffin, Donald R., Harvard University, Cambridge, Massachusetts

Guyselman, J. Bruce, Carleton College, Northfield, Minnesota

Halberg, Erna, University of Minnesota Medical School, Minneapolis, Minnesota

Halberg, Franz, University of Minnesota Medical School, Minneapolis, Minnesota

Hamicton, William J., California Academy of Sciences, San Francisco, California

Hamner, KaRL C., University of California, Los Angeles, California

HAN, JACK, State University of New York, Syracuse, New York 
Harker, Janet E., Zoological Laboratory, Cambridge, England

Hasler, Arthur D., University of Wisconsin, Madison, Wisconsin

Hastings, Antolnette, American Association for the Advancement of Science, Westbury, New York

Hastings, J. Wooduand, University of Illinois, Urbana, Illinois

Hauenschild, C., Zoologisches Institut der Universität, Freiburg/Br., Germany

Hayes, Helen, Office of Naval Research, Washington, D. C.

HellbrügGe, Theodor, Kinderpoliklinik der Universität, München, München, Germany

Helms, Carl W., Massachusetts Audubon Society, South Lincoln, Massachusetts

Hendricks, Sterling B., U. S. Department of Agriculture, Beltsville, Maryland

Highkin, H. R., California Institute of Technology, Pasadena, California

Hills, Alicia, Life Magazine, New York City

Hoagland, Hudson, The Worcester Foundation for Experimental Biology, Shrewsbury, Massachusetts

Hoffmann, Klaus, Max-Planck-Institut für Verhaltensphysiologie, Erling-Andechs/Obb., Germany, and Princeton Lniversity, Princeton, New Jersey

Hopwoon, Joseph, Colorado State University, Fort Collins, Colorado

Hubbard, Henry, Newsweek, New York City

IsaAc, Donald, University of California, Berkeley, California

JACobs, Gforge, National Aeronautics and Space Administration, Washington, D. C.

Jenner, Charles E., University of North Carolina, Chapel Hill, North Carolina

Kahn, David, Newsday, Garden City, New York

Kalmus, Hans, University College London, London, England

Karakashian, Marlene W., University of California, Los Angeles, California

Kato, Masaru, Washington State University, Pullman, Washington

Kaufmann, Berwind N., University of Mississippi Medical Center, Jackson, Mississippi

Kaufmann, Berwind P., Carnegie Institution of Washington, Cold Spring Harbor, New York

Kennedy, Donald, Syracuse University, Syracuse, New York

Kernaghan, Roy Peter, Biological Laboratory, Cold Spring Harbor, New York

KLotTer, KaRL, Institut für Angewandte Mechanik, Darmstadt, Germany

Kramer, Sol, State University College on Long Island, Oyster Bay, New York

Krauss, Marian, Biological Laboratory, Cold Spring Harbor, New York
Kris, E. Christine, Cambridge, Massachusetts

Larsen, Victor, Adelphi College, Garden City, New York

Lees, Anthony D., Agricultural Research Council, Cambridge, England

Lehrman, Daniel S., Rutgers University, Newark, New Jersey

Leinwhibr, Franz J., Johns Hopkins University, Baltimore, Maryland

LEINWEBER, Lupe, Baltimore, Maryland

Lienau, Carl, Columbia University, New York City

Lincoln, Richard G., Long Beach State College, Long Beach, California

Lindauer, Martin, Zoologisches Institut der Universität München, München, Germany

Lobban, Mary C., Medical Research Council Laboratories, London, England

MacDoweld, E. C., Biological Laboratory, Cold Spring Harbor, New York

Malm, Mrgnon, Princeton University, Princeton, New Jersey

Marshall, 'A. J., Monash University, Victoria, Australia

Menaker, Michael, Harvard University, Cambridge, Massachusetts

Mrrcer, Derwent M. A., Physical Laboratory, The University, Southampton, England

Millman, Edward, Grumman Aireraft Engineering Corporation, Bethpage, New York

Minis, Dorothy, Princeton University, Princeton, New Jersey

Mirake, Akio, Osaka City University, Osaka, Japan

Moe, Gordon K., Masonic Medical Research Laboratory, Utica, New York

Morr, Syurt, Zoological Institute, Kyoto University, Kyoto, Japan

MukaI, Frank, Carnegie Institution of Washington, Cold Spring Harbor, New York

Novick, Alvin, Yale University, New Haven, Connecticut

Nowosielski, Jozer, Cornell University, Ithaca, New York

Oftтing, Bonnalie J., Northwestern University, Evanston, Illinois

OLtve, John R., American Institute of Biological Sciences, Washington, D. C.

Palmer, John, Northwestern University, Evanston, Illinois

Petropulos, Stephen F., Princeton University, Princeton, New Jersey

Pittendrigh, Colin S., Princeton University, Princeton, New Jersey

Prokop, Barbara, Biological Laboratory, Cold Spring Harbor, New York

Rawson, Kenneth S., Swarthmore College, Swarthmore, Pennsylvania 
Rawson, Kenneth S., Mrs., Swarthmore College, Swarthmore, Pennsylvania

ReNNeR, MAx, Zoologisches Institut der Universität, München, Germany

Richter, Curt P., The Johns Hopkins Hospital, Baltimore, Maryland

Roberts, Shepherd K., Princeton University, Princeton, New Jersey

Sandeen, Muriel I., Duke University, Durham, North Carolina

Savbly, Harvey E., U. S. Air Force, Washington, D. C.

Schalet, Ruth, Biological Laboratory, Cold Spring Harbor, New York

Scharrer, BerTa, Albert Einstein College of Medicine, New York City

Scharrer, Ernst, Albert Einstein College of Medicine, New York City

Scheele, George A., Princeton University, Princeton, New Jersey

Schefano, Anthony, Hofstra College, Hempstead, New York

Schmidt-Koenig, Klaus, Max-Planck-Institut für Verhaltensphysiologie, Wilhelmshaven, Germany, and Duke University, Durham, North Carolina

Sснмiтт, Отто H., University of Minnesota, Minneapolis, Minnesota

Sснмітт, Отто H., Mrs., University of Minnesota, Minneapolis, Minnesota

Schwassmann, Horst O., University of Wisconsin, Madison, Wisconsin

Schwemmle, Berthold, Pflanzenphysiologisches Institut der Universität, Göttingen, Germany

SEgal, EarL, Kansas State Teachers College, Emporia, Kansas

Shapiro, Arthur, New York State University, Brooklyn, New York

Shapiro, Seymour, Brookhaven National Laboratory, Upton, New York

Simon, Edward, Carnegie Institution of Washington, Cold Spring Harbor, New York

Sinohi, G. S., University of California, Los Angeles, California
Smolker, Robert, State University of New York, Oyster Bay, New York

Sokoloff, Alexander, William H. Miner Agricultural Research Institute, Chazy, New York

Sokoloff, Alexander, Mrs., William H. Miner Agricultural Research Institute, Chazy, New York

Sollberger, ArNe, Caroline Institute, Stockholm, Sweden

Suckling, Eustace, State University of New York Medical College, Brooklyn, New York

Swade, Rrchard H., Princeton University, Princeton, New Jersey

Sweeney, Beatrice M., Lniversity of California, LaJolla, California

Tall, Benita, American Institute of Biological Sciences, Washington, D. C.

Thumann, Kenneth V., Harvard University, Cambridge, Massachusetts

Thornburg, Wayne, Dartmouth Medical School, Hanover, New Hampshire

TOLLES, WALTER, Institute of Oceanography and Marine Biology, Oyster Bay, New York

Wallraff, Hans G., Max-Planck-Institut für Verhaltensphysiologie, Wilhelmshaven, Germany

Webb, H. Marguerite, Stazione Zoologica, Napoli, Italy

Weitzman, Mary, Albert Einstein College of Medicine, New York City

Welds, G. P., University College London, London, England

Went, F. W., Missouri Botanical Garden, St. Louis, Missouri

Wever, RütGer, Max-Planck-Institut für Verhaltensphysiologie, Erling-Andechs/Obb, Germany

Wilder, JosePh, Society for Biological Rhythms, Stockholm, Sweden

Wilkins, Malcolm B., University of London King's College, London, England

Wisby, Warren, University of Miami, Miami, Florida

Wolf, William, New York City

Wolfson, Albert, Northwestern University, Evanston, Illinois

Wülff, Verner J., Masonic Medical Research Laboratory, Utica, New York 


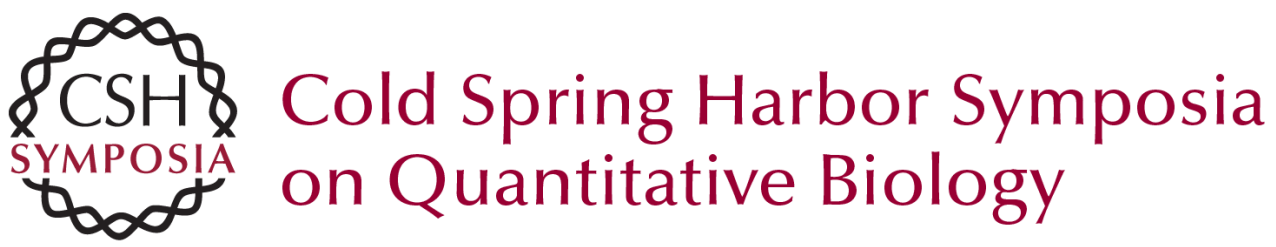

\section{List of Those Attending the Symposium}

Cold Spring Harb Symp Quant Biol 1960 25: ix-xi

Access the most recent version at doi:10.1101/SQB.1960.025.01.002

\section{License}

Email Alerting Receive free email alerts when new articles cite this article - sign up in Service the box at the top right corner of the article or click here. 